\title{
Predictive significance of standardized uptake value parameters of FDG-PET in patients with non-small cell lung carcinoma
}

\author{
X.-Y. Duan, W. Wang, M. Li, Y. Li and Y.-M. Guo
}

PET-CT Center, The First Affiliated Hospital of Xi'an, Jiaotong University, Xi'an, Shaanxi, China

\begin{abstract}
${ }^{18} \mathrm{~F}$-fluoro-2-deoxyglucose (FDG) positron emission tomography (PET)/computed tomography (CT) is widely used to diagnose and stage non-small cell lung cancer (NSCLC). The aim of this retrospective study was to evaluate the predictive ability of different FDG standardized uptake values (SUVs) in 74 patients with newly diagnosed NSCLC. ${ }^{18}$ F-FDG PET/CT Scans were performed and different SUV parameters $\left(S U V_{\text {max }}, S U V_{a v g}, S U V_{T / L}\right.$, and $\left.S U V_{T / A}\right)$ obtained, and their relationship with clinical characteristics were investigated. Meanwhile, correlation and multiple stepwise regression analyses were performed to determine the primary predictor of SUVs for NSCLC. Age, gender, and tumor size significantly affected SUV parameters. The mean SUVs of squamous cell carcinoma were higher than those of adenocarcinoma. Poorly differentiated tumors exhibited higher SUVs than well-differentiated ones. Further analyses based on the pathologic type revealed that the SUV max, SUV $V_{a v g}$ and SUV $V_{T / L}$ of poorly differentiated adenocarcinoma tumors were higher than those of moderately or well-differentiated tumors. Among these four SUV parameters, SUV $\mathrm{T}_{\mathrm{T} / \mathrm{L}}$ was the primary predictor for tumor differentiation. However, in adenocarcinoma, SUV $_{\max }$ was the determining factor for tumor differentiation. Our results showed that these four SUV parameters had predictive significance related to NSCLC tumor differentiation; SUV $\mathrm{T/L}$ appeared to be most useful overall, but SUV $\max$ was the best index for adenocarcinoma tumor differentiation.
\end{abstract}

Key words: ${ }^{18}$ F-FDG PET/CT; Standardized uptake value (SUV); Prediction; Non-small cell lung cancer

\section{Introduction}

Lung cancer is the largest contributor to cancer death worldwide (1). ${ }^{18} \mathrm{~F}$-fluoro-2-deoxyglucose $\left({ }^{18} \mathrm{~F}\right.$-FDG) positron emission tomography (PET) has been widely used in the diagnosis and staging of non-small cell lung cancer (NSCLC) (2,3). Increased FDG uptake by lung cancer cells, measured as the maximum standardized uptake value $\left(S U V_{\text {max }}\right)$, has been reported to predict the biologic aggressiveness of both early and advanced NSCLC (4-10); however, the reliability of SUV $V_{\text {max }}$ remains controversial. Some studies found no correlation between $S \mathrm{SV}_{\max }$ and patient prognosis or lung cancer aggressiveness $(11,12)$. Reasonable explanations for this include the following: 1) SUV $_{\text {max }}$ reportedly varies among PET scanners (13); 2) uncontrolled factors such as glucose level, duration of the uptake period, body weight, body composition, and recovery coefficient introduce considerable variations in $\operatorname{SUV}_{\max }(14)$; 3) different acquisition and image reconstruction parameters also affect $S U V_{\max }(15)$. Recent studies have shown that the average SUV (SUV $\left.{ }_{a v g}\right)$, ratio of tumor SUV $_{\text {max }}$ to liver SUV $\left(S U V_{T / L}\right)$, and the ratio of tumor
SUV $_{\max }$ to the blood pool SUV of aorta $\left(\mathrm{SUV}_{\mathrm{T} / \mathrm{A}}\right)$ could provide better predictive values $(16,17)$. The purpose of this study was to assess the predictive significance of these SUV parameters in patients with newly diagnosed NSCLC.

\section{Patients and Methods}

\section{Study population}

This was a retrospective study. Seventy-four consecutive NSCLC patients who were histologically diagnosed between April 2011 and December 2012 were included in this study. They had not undergone surgery, chemotherapy, or radiation therapy and did not have extensive liver metastases. All patients in the study received integrated PET/CT scans with the same PET/CT system within 1 week before surgery or biopsy. The tumor-node-metastasis (TNM) staging system was used, and the histologic tumor type was categorized according to the World Health Organization (WHO) classification system $(18,19)$. The study was

Correspondence: Xiao-Yi Duan: <duanxy@mail.xjtu.edu.cn>.

Received June 5, 2014. Accepted October 22, 2014. First published online February 3, 2015. 
Table 1. Baseline characteristics and standardized uptake values (SUVs) of the study population $(n=74)$.

\begin{tabular}{|c|c|c|c|c|c|}
\hline Characteristics & Number & $S U V_{\max }$ & SUV $_{\text {avg }}$ & $S U V_{T / L}$ & $\mathrm{SUV}_{\mathrm{T} / \mathrm{A}}$ \\
\hline \multicolumn{6}{|l|}{ Age } \\
\hline$<60$ years & 32 & $6.9 \pm 3.0$ & $5.7 \pm 2.4$ & $4.0 \pm 2.1$ & $4.5 \pm 2.2$ \\
\hline$\geqslant 60$ years & 42 & $9.1 \pm 4.8$ & $7.2 \pm 3.7$ & $5.0 \pm 2.5$ & $5.9 \pm 3.1$ \\
\hline $\mathrm{P}$ & & $0.022^{*}$ & $0.041^{*}$ & 0.063 & $0.027^{*}$ \\
\hline \multicolumn{6}{|l|}{ Gender } \\
\hline Male & 54 & $8.7 \pm 4.3$ & $6.9 \pm 3.2$ & $4.9 \pm 2.4$ & $5.4 \pm 2.9$ \\
\hline Female & 20 & $6.6 \pm 3.8$ & $5.7 \pm 3.4$ & $3.5 \pm 2.0$ & $4.3 \pm 2.3$ \\
\hline$P$ & & 0.053 & 0.189 & $0.018^{*}$ & $0.041^{*}$ \\
\hline \multicolumn{6}{|l|}{ Tumor size } \\
\hline$<3 \mathrm{~cm}$ & 35 & $5.8 \pm 2.8$ & $4.8 \pm 2.2$ & $3.5 \pm 2.2$ & $3.9 \pm 2.1$ \\
\hline$\geqslant 3 \mathrm{~cm}$ & 39 & $10.3 \pm 4.3$ & $8.2 \pm 3.2$ & $5.4 \pm 2.2$ & $6.6 \pm 2.7$ \\
\hline $\mathrm{P}$ & & $0.000^{*}$ & $0.000^{*}$ & $0.000^{*}$ & $0.000^{*}$ \\
\hline \multicolumn{6}{|l|}{ Pathological type } \\
\hline Adenocarcinoma & 50 & $7.4 \pm 3.9$ & $5.9 \pm 2.9$ & $4.0 \pm 2.2$ & $4.8 \pm 2.8$ \\
\hline Squamous cell carcinoma & 24 & $9.8 \pm 4.6$ & $7.9 \pm 3.6$ & $5.5 \pm 2.5$ & $6.3 \pm 2.6$ \\
\hline$P$ & & $0.035^{\star}$ & $0.025^{*}$ & $0.017^{*}$ & $0.032^{*}$ \\
\hline \multicolumn{6}{|l|}{ Tumor differentiation } \\
\hline Well & 15 & $5.7 \pm 2.8$ & $4.7 \pm 2.4$ & $2.9 \pm 1.5$ & $3.5 \pm 1.7$ \\
\hline Moderate & 22 & $8.0 \pm 3.9$ & $6.7 \pm 3.4$ & $4.3 \pm 2.2$ & $5.2 \pm 2.5$ \\
\hline Poor & 37 & $9.2 \pm 4.6$ & $7.2 \pm 3.3$ & $5.3 \pm 2.5$ & $6.1 \pm 3.0$ \\
\hline $\mathrm{P}$ & & $0.027^{\star}$ & $0.039^{*}$ & $0.004^{*}$ & $0.010^{*}$ \\
\hline \multicolumn{6}{|l|}{ Clinical stage } \\
\hline I & 12 & $7.1 \pm 5.5$ & $5.7 \pm 4.6$ & $3.5 \pm 2.7$ & $4.0 \pm 2.9$ \\
\hline II & 9 & $8.3 \pm 3.7$ & $6.4 \pm 1.8$ & $4.8 \pm 1.5$ & $6.3 \pm 3.1$ \\
\hline III & 23 & $8.3 \pm 3.4$ & $6.6 \pm 2.6$ & $4.8 \pm 2.1$ & $5.5 \pm 2.2$ \\
\hline IV & 30 & $8.4 \pm 4.6$ & $6.9 \pm 3.5$ & $4.6 \pm 2.6$ & $5.4 \pm 3.0$ \\
\hline $\mathrm{P}$ & & 0.835 & 0.757 & 0.409 & 0.274 \\
\hline
\end{tabular}

Data are reported as means $\pm S D$. SUV $\max :$ maximum SUV; SUV ${ }_{\text {avg: }}$ average SUV; SUV $V_{T / L}$ : ratio of tumor SUV $V_{\max }$ to liver SUV; SUV ratio of tumor SUV max to the blood pool SUV of aorta. One-way ANOVA followed by the LSD post hoc test was used for statistical analyses.
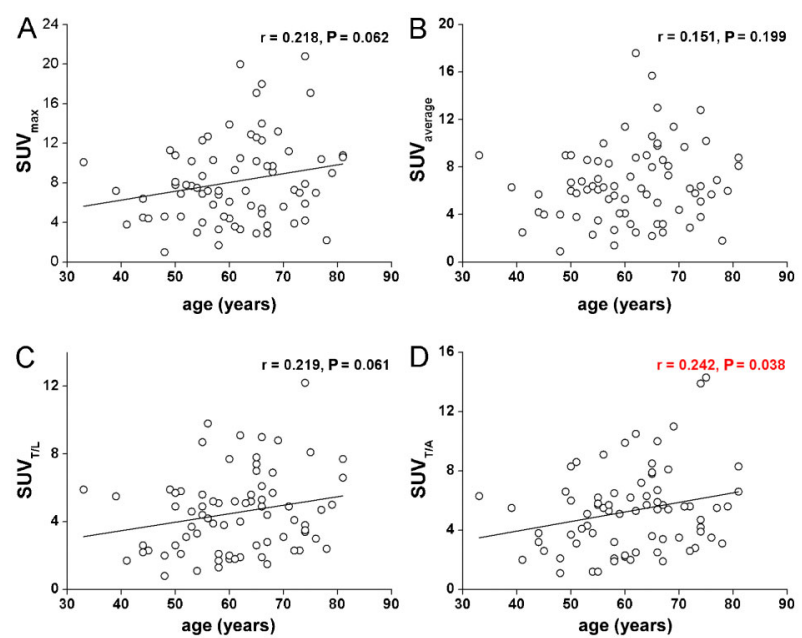

Figure 1. Correlation analyses between age and standardized uptake values (SUVs). SUV max: maximum SUV; SUV ${ }_{\text {avg: average }}$ SUV; SUV $V_{T / L}$ : ratio of tumor SUV $\max$ to liver SUV; SUV $V_{T / A}:$ ratio of tumor SUV max $_{\text {max }}$ to the blood pool SUV of aorta.
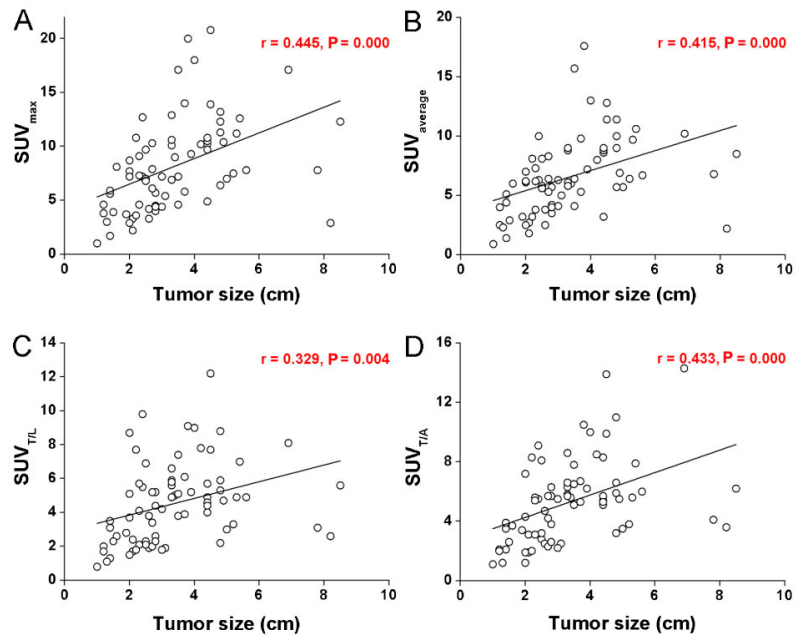

Figure 2. Correlation analyses between tumor size and standardized uptake values (SUVS). SUV max: maximum SUV; $S U V_{\text {avg }}$ : average SUV; $S U V_{T / L}$ : ratio of tumor $S U V_{\max }$ to liver SUV; SUV $V_{T / A}$ : ratio of tumor SUV $V_{\text {max }}$ to the blood pool SUV of aorta. 

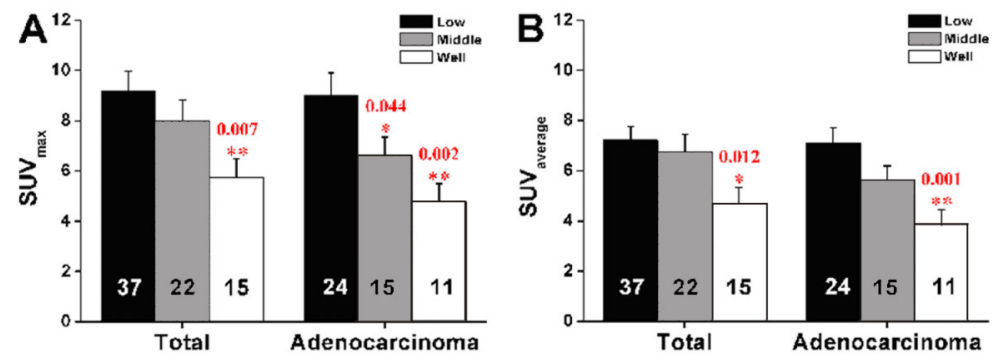

Figure 3. Tumor differentiation-based group difference for standardized uptake values (SUVs). One-way ANOVA followed by the LSD post hoc test was used for statistical analyses. See Figure 1 for explanation of SUVs.
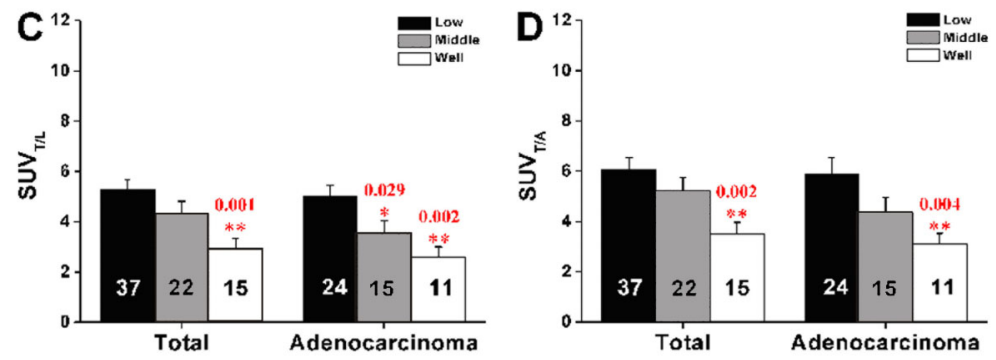

conducted with the approval of the Institutional Ethics Committee of Xi'an Jiaotong University.

\section{PET/CT scan}

Patients were asked to fast for at least $6 \mathrm{~h}$ before examination, and serum glucose levels were confirmed to be below $160 \mathrm{mg} / \mathrm{dL}$. PET/CT scanning was performed on a Gemini 64 TF scanner (Philips, The Netherlands) 4060 min after intravenous FDG administration (3.7-4.4 MBq/ $\mathrm{kg})$. Non-contrast CT images were obtained with a multidetector spiral CT scanner (Philips Gemini TF 16 PET/CT) immediately prior to PET scanning with an acquisition time of $1.5 \mathrm{~min} / \mathrm{bed}$ position during shallow breathing. The scan field was from the vertex to the upper thighs. PET data were reconstructed using an ordered-subset expectation maximization algorithm. CT data were used for attenuation correction and anatomic localization. Co-registered images were displayed by means of the SYNTEGRA software (Philips).

$\mathrm{PET} / \mathrm{CT}$ results were interpreted by two experienced nuclear medicine physicians in a blinded manner. SUV $V_{\text {max }}$ and SUV $\mathrm{Vuv}_{\text {avg }}$ were determined by drawing a region of interest (ROI) around the primary tumor on the transaxial slices and calculating values with the following equation: tumor activ- ity concentration/injected dose/body weight. $S U V_{T / L}$ and SUV $_{\mathrm{T} / \mathrm{A}}$ were defined as primary tumor SUV $_{\max }$ divided by liver SUV $V_{\text {max }}$ and aorta blood pool SUV $V_{\text {max }}$, respectively.

\section{Statistical analysis}

All analyses were conducted using the SPSS software package (version 18.0, SPSS Inc., USA). The statistical differences of SUVs among the groups were determined using one-way analysis of variance (ANOVA), and LSD post hoc testing was performed to determine the specific differences between the two groups when $\mathrm{P}<0.05$. Multiple stepwise regression analyses were applied to test the merit of SUVs to predict NSCLC outcomes. Differences were considered significant when $\mathrm{P}<0.05$.

\section{Results}

From April 2011 through December 2012, a total of 74 consecutive patients with a mean age of $61 \pm 10$ years ( median $=62$ years; range $=33-81$ years) were enrolled in the study. The median SUV $_{\max }$ was 7.25 (range= 1.0-20.8), and the median SUV $_{\text {avg }}$ was 6.2 (range $=0.9$ 17.6). The median $S U V_{T / L}$ and $S U V_{T / A}$ were 4.4 (range $=$ $0.8-12.2$ ) and 5.4 (range $=1.2-14.3$ ), respectively. The

Table 2. Multiple stepwise regression analysis in patients with non-small cell lung cancer $(n=74)$.

\begin{tabular}{|c|c|c|c|c|c|}
\hline & \multicolumn{2}{|c|}{ Unstandardized coefficients } & \multirow{2}{*}{$\frac{\text { Standardized coefficients }}{\text { Beta }}$} & \multirow[t]{2}{*}{$t$} & \multirow[t]{2}{*}{$\mathrm{P}$} \\
\hline & B & Std. error & & & \\
\hline$S U V_{T / L}$ & 0.117 & 0.037 & 0.346 & 3.134 & $0.002^{*}$ \\
\hline
\end{tabular}

Dependent variable: tumor differentiation degree. $S_{U} V_{T / L}$ : ratio of tumor maximum standardized uptake value $\left(S U V_{\max }\right)$ to liver SUV. 

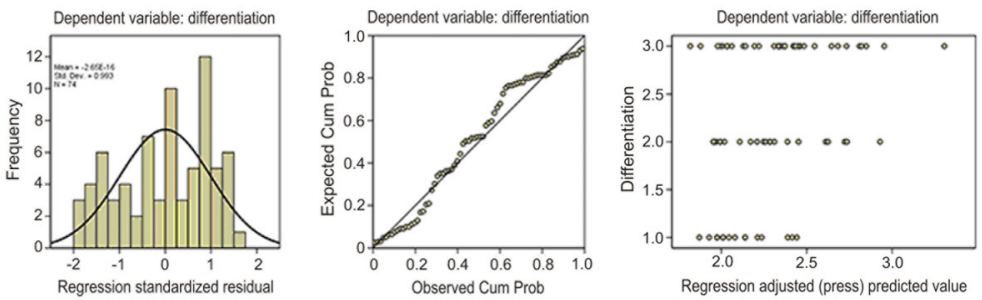

Figure 4. Multiple stepwise regression analysis of patients with non-small cell lung cancer (NSCLC).

demographic and clinical characteristics and SUVs of the population are summarized in Table 1. The mean $S U V_{T / L}$ and $\mathrm{SUV}_{\mathrm{T} / \mathrm{A}}$ of males were significantly higher than those of female subjects $(P=0.018,0.041)$. Significantly higher $S U V_{\text {max }}, S U V_{\text {avg }}$, and SUV $V_{T / A}$ were observed in patients $\geq 60$ years of age compared to those $<60$ years $(P=0.022$, 0.041 , and 0.027 , respectively). Only $S U V_{T / A}$ positively correlated with patients' age $(r=0.242, \mathrm{P}=0.038$; Figure 1). No significant relationships were observed between different SUV parameters and clinical stages (all $P>0.05$, Table 1).

Tumor size was an important factor related to SUV on PET. The median size of the primary tumor was $3.05 \mathrm{~cm}$ (range $=1.0-8.5 \mathrm{~cm}$ ), and the mean SUVs of tumors $\geq 3 \mathrm{~cm}$ were statistically higher than those $<3 \mathrm{~cm}(P=0.000$, Table 1). Further analysis revealed that SUVs significantly correlated with tumor size. Their values increased in a tumor size-dependent manner (Figure 2).

Table 1 shows that tumor pathologic type and differentiated grade were significantly related to $S U V_{\max }(P=$ 0.035 and 0.027$),$ SUV $_{\text {avg }}(P=0.025$ and 0.039$), S_{\mathrm{SU}}$ $(P=0.017$ and 0.004$)$ and $S_{U} V_{T / A}(P=0.032$ and 0.010$)$. Poorly differentiated tumors exhibited higher SUVs than well-differentiated tumors $(P<0.05$, Figure 3$)$. We performed further analyses based on pathologic type and found that in patients with adenocarcinoma, $S_{\text {max }}(P=$ 0.005), SUV avg $(P=0.007)$, and $\operatorname{SUV}_{\mathrm{T} / \mathrm{L}}(P=0.018)$ were significantly different among the differentiation groups. Specifically, the values of poorly differentiated tumors were statistically higher than those of moderately or welldifferentiated tumors ( $P<0.05$, Figure 3$)$.

Multiple stepwise regression analyses revealed that of the four SUV parameters, $S_{\mathrm{T} / \mathrm{L}}$ was the primary predictor for tumor differentiation (Table 2 and Figure 4), while in patients with adenocarcinoma, SUV $V_{\max }$ was the best independent factor for determining tumor differentiation (Table 3 and Figure 5).

\section{Discussion}

SUV is a semi-quantitative index of radiolabeled glucose uptake in tumor tissue and correlates with some prognostic factors, including tumor differentiation (20). SUV max $_{\text {mas }}$ been reported to relate to tumor grade, clinical stage, and pathologic type (21). In the present study, we also noted a higher SUV $_{\max }$ in squamous cell carcinoma than in adenocarcinoma, and found that poorly differentiated tumors showed a higher SUV $V_{\max }$ than well-differentiated tumors. The same results were obtained for SUV avg

In clinical practice, some uncontrolled factors affect SUV, such as blood glucose level, the time window between FDG administration and image acquisition, serum insulin levels, and renal clearance of FDG $(22,23)$. As such, the use of $S U V_{T / L}$ and $S U V_{T / A}$ has been proposed to minimize variability. Shiono et al. (17) reported that these ratios could significantly predict the risk of disease recurrence in patients with lung cancer, but no more data were published to support this concept. The present study enrolled 74 newly diagnosed NSCLC patients and applied the same method to obtain the SUV ratios $\left(\mathrm{SUV}_{\mathrm{T} / \mathrm{L}}\right.$ and $\left.S U V_{\mathrm{T} / \mathrm{A}}\right)$ for each patient. Statistical analyses demonstrated that both values correlated with tumor differentiation. Specifically, poorly differentiated tumors had higher SUV ratios than well-differentiated tumors. Further multiple stepwise regression analysis showed that among these parameters $\left(S U V_{\text {max }}, S U V_{\text {avg }}, S U V_{T / L}\right.$, and $\left.S U V_{T / A}\right), S U V_{T / L}$ was an independent determinant for tumor differentiation in NSCLC patients. However, none of these parameters were found to be useful in predicting NSCLC clinical stage.

Pathologic type was related to SUVs. In our study, squamous cell carcinomas exhibited significantly higher SUVS than adenocarcinomas. Conversely, SUV max, $\mathrm{SUV}_{\text {avg }}$, and $\mathrm{SUV}_{\mathrm{T} / \mathrm{L}}$ correlated with tumor differentiation in adenocarcinoma, and SUV $V_{\max }$ was the best independent

Table 3. Multiple stepwise regression analysis in patients with lung adenocarcinoma $(n=50)$.

\begin{tabular}{|c|c|c|c|c|c|}
\hline & \multicolumn{2}{|c|}{ Unstandardized coefficients } & \multirow{2}{*}{$\frac{\text { Standardized coefficients }}{\text { Beta }}$} & \multirow[t]{2}{*}{$t$} & \multirow[t]{2}{*}{$P$} \\
\hline & B & Std. error & & & \\
\hline $\mathrm{SUV}_{\mathrm{T} / \mathrm{L}}$ & 0.094 & 0.027 & 0.443 & 3.428 & $0.001^{*}$ \\
\hline
\end{tabular}

Dependent variable: tumor differentiation degree. $S U V_{T / L}$ : ratio of tumor maximum standardized uptake value $\left(S U V_{\max }\right)$ to liver SUV. 

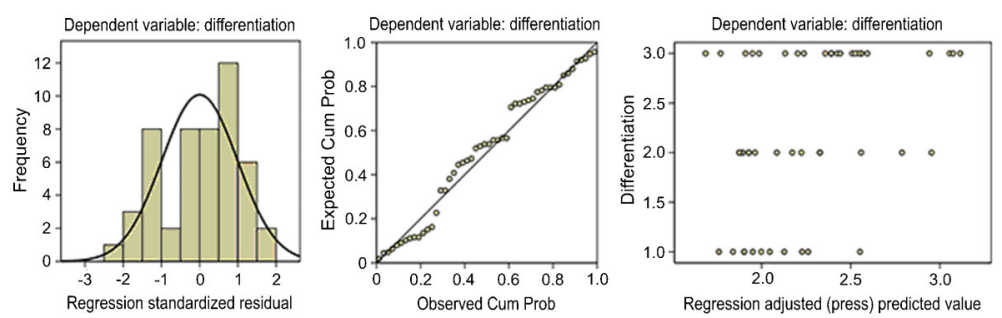

Figure 5. Multiple stepwise regression analysis in patients with lung adenocarcinoma.

determining factor for tumor differentiation, which was inconsistent with the result in the entire study population. Further investigation is needed to understand this finding.

Furthermore, tumor size, age, and gender were also associated with differences in SUV parameters. The values were increased for larger tumors, and patients $\geq 60$ years of age had higher values than those $<60$ years. We also observed statistical differences in SUV ratios between male and female subjects. Males had significantly higher $S U V_{T / L}$ and $S U V_{T / A}$ ratios than females. More data are required to confirm these findings.

Based on the results above, we came to the following preliminary conclusions: 1) $\mathrm{SUV}_{\max }, \mathrm{SUV}_{\mathrm{avg}}, \mathrm{SUV}_{\mathrm{T} / \mathrm{L}}$, and $\mathrm{SUV}_{\mathrm{T} / \mathrm{A}}$ relate to tumor differential grade and might be useful for predicting NSCLC patient prognosis; 2) of these parameters, SUV $\mathrm{V}_{\mathrm{T} / \mathrm{L}}$ exhibited the strongest predictive

\section{References}

1. Youlden DR, Cramb SM, Baade PD. The International Epidemiology of Lung Cancer: geographical distribution and secular trends. J Thorac Oncol 2008; 3: 819-831, doi: 10.1097/ JTO.0b013e31818020eb.

2. Pieterman RM, van Putten JW, Meuzelaar JJ, Mooyaart EL, Vaalburg W, Koeter GH, et al. Preoperative staging of nonsmall-cell lung cancer with positron-emission tomography. $N$ Engl J Med 2000; 343: 254-261, doi: 10.1056/NEJM200007 273430404.

3. Toloza EM, Harpole L, McCrory DC. Noninvasive staging of non-small cell lung cancer: a review of the current evidence. Chest 2003; 123: 137S-146S, doi: 10.1378/chest.123.1_ suppl.137S

4. Vansteenkiste JF, Stroobants SG, Dupont PJ, De Leyn PR, Verbeken EK, Deneffe GJ, et al. Prognostic importance of the standardized uptake value on (18)F-fluoro-2-deoxyglucose-positron emission tomography scan in non-smallcell lung cancer: An analysis of 125 cases. Leuven Lung Cancer Group. J Clin Oncol 1999; 17: 3201-3206.

5. Higashi K, Ueda Y, Arisaka Y, Sakuma T, Nambu Y, Oguchi $\mathrm{M}$, et al. ${ }^{18} \mathrm{~F}-\mathrm{FDG}$ uptake as a biologic prognostic factor for recurrence in patients with surgically resected non-small cell lung cancer. J Nucl Med 2002; 43: 39-45.

6. Downey RJ, Akhurst T, Gonen M, Vincent A, Bains MS, Larson S, et al. Preoperative F-18 fluorodeoxyglucosepositron emission tomography maximal standardized uptake value predicts survival after lung cancer resection. J Clin Oncol 2004; 22: 3255-3260, doi: 10.1200/JCO.2004.11.109.

7. Cerfolio RJ, Bryant AS, Ohja B, Bartolucci AA. The value for tumor differentiation overall, but SUV $_{\max }$ was better than other parameters for predicting lung adenocarcinoma differentiation; and 3) age, gender, tumor size, and pathologic type dramatically affected SUV parameters and should therefore be taken into account during imaging interpretation. Because of the limitations associated with retrospective studies, further prospective investigations should be designed and performed to acquire more data on the prognostic significance of different SUV parameters in NSCLC patients.

\section{Acknowledgments}

The authors acknowledge the work of all investigators involved in this study. This research was supported by the Natural Science Foundation of China (\#81171397). maximum standardized uptake values on positron emission tomography of a non-small cell lung cancer predict stage, recurrence, and survival. J Thorac Cardiovasc Surg 2005; 130: 151-159, doi: 10.1016/j.jtcvs.2004.11.007.

8. Al-Sarraf N, Gately K, Lucey J, Aziz R, Doddakula K, Wilson $\mathrm{L}$, et al. Clinical implication and prognostic significance of standardised uptake value of primary non-small cell lung cancer on positron emission tomography: analysis of 176 cases. Eur J Cardiothorac Surg 2008; 34: 892-897, doi: 10.1016/j.ejcts.2008.07.023.

9. Maeda R, Isowa $\mathrm{N}$, Onuma $\mathrm{H}$, Miura $\mathrm{H}$, Harada $\mathrm{T}$, Touge $\mathrm{H}$, et al. The maximum standardized $18 \mathrm{~F}$-fluorodeoxyglucose uptake on positron emission tomography predicts lymph node metastasis and invasiveness in clinical stage IA nonsmall cell lung cancer. Interact Cardiovasc Thorac Surg 2009; 9: 79-82, doi: 10.1510/icvts.2008.201251.

10. Paesmans M, Berghmans T, Dusart M, Garcia C, HosseinFoucher C, Lafitte JJ, et al. Primary tumor standardized uptake value measured on fluorodeoxyglucose positron emission tomography is of prognostic value for survival in non-small cell lung cancer: update of a systematic review and meta-analysis by the European Lung Cancer Working Party for the International Association for the Study of Lung Cancer Staging Project. J Thorac Oncol 2010; 5: 612-619.

11. Vesselle H, Freeman JD, Wiens L, Stern J, Nguyen HQ, Hawes SE, et al. Fluorodeoxyglucose uptake of primary nonsmall cell lung cancer at positron emission tomography: new contrary data on prognostic role. Clin Cancer Res 2007; 13: 3255-3263, doi: 10.1158/1078-0432.CCR-06-1128. 
12. Downey RJ, Akhurst T, Gonen M, Park B, Rusch V. Fluorine18 fluorodeoxyglucose positron emission tomographic maximal standardized uptake value predicts survival independent of clinical but not pathologic TNM staging of resected nonsmall cell lung cancer. J Thorac Cardiovasc Surg 2007; 133: 1419-1427, doi: 10.1016/j.jtcvs.2007.01.041.

13. Bryant AS, Cerfolio RJ, Klemm KM, Ojha B. Maximum standard uptake value of mediastinal lymph nodes on integrated FDG-PET-CT predicts pathology in patients with non-small cell lung cancer. Ann Thorac Surg 2006; 82: 417422, doi: 10.1016/j.athoracsur.2005.12.047.

14. Keyes JW Jr. SUV: standard uptake or silly useless value? J Nucl Med 1995; 36: 1836-1839.

15. Ivanovic $M$, Loncaric $S$, McCartney $\mathrm{WH}$, Khandani $A$. Evaluation of PET standard uptake values dependence on imaging protocols for dual time point imaging. IEEE 2004; 7 : 4076-4079.

16. Kanstrup IL, Klausen TL, Bojsen-Moller J, Magnusson P, Zerahn B. Variability and reproducibility of hepatic FDG uptake measured as SUV as well as tissue-to-blood background ratio using positron emission tomography in healthy humans. Clin Physiol Funct Imaging 2009; 29: 108113, doi: 10.1111/j.1475-097X.2008.00846.x.

17. Shiono S, Abiko M, Okazaki T, Chiba M, Yabuki H, Sato T. Positron emission tomography for predicting recurrence in stage I lung adenocarcinoma: standardized uptake value corrected by mean liver standardized uptake value. Eur $\mathrm{J}$ Cardiothorac Surg 2011; 40: 1165-1169.

18. Beasley MB, Brambilla E, Travis WD. The 2004 World Health Organization classification of lung tumors. Semin Roentgenol 2005; 40: 90-97, doi: 10.1053/j.ro.2005.01. 001.

19. Detterbeck FC, Boffa DJ, Tanoue LT. The new lung cancer staging system. Chest 2009; 136: 260-271, doi: 10.1378/ chest.08-0978.

20. Chen JC, Huang TW, Cheng YL, Chang H, Tzao C, Huang WS, et al. Prognostic value of 18-FDG uptake in early stage NSCLC. Thorac Cardiovasc Surg 2009; 57: 413-416, doi: 10.1055/s-0029-1185733.

21. Lu P, Yu L, Li Y, Sun Y. A correlation study between maximum standardized uptake values and pathology and clinical staging in nonsmall cell lung cancer. Nucl Med Commun 2010; 31: 646-651.

22. Kubota $\mathrm{K}$, Watanabe $\mathrm{H}$, Murata $\mathrm{Y}$, Yukihiro $\mathrm{M}$, Ito $\mathrm{K}$, Morooka $M$, et al. Effects of blood glucose level on FDG uptake by liver: a FDG-PET/CT study. Nucl Med Biol 2011; 38: 347-351, doi: 10.1016/j.nucmedbio.2010.09.004.

23. Boellaard R, Oyen WJ, Hoekstra CJ, Hoekstra OS, Visser EP, Willemsen AT, et al. The Netherlands protocol for standardisation and quantification of FDG whole body PET studies in multi-centre trials. Eur J Nucl Med Mol Imaging 2008; 35: 2320-2333, doi: 10.1007/s00259-008-0874-2. 